\title{
Correction to: Unicity on Entire Function Concerning Its Differential-Difference Operators
}

\author{
XiaoHuang Huang(1)
}

\begin{abstract}
In this paper, we give a correction to Theorem 1 in a previous paper [Results Math. 76, Article number: 147 (2021)].
\end{abstract}

Mathematics Subject Classification. 30D35, 39A70.

Keywords. Uniqueness, entire functions, differential-differences operators.

\section{Correction to: Results Math (2021) 76:147 https://doi.org/10.1007/s00025-021-01461-y}

\section{Introduction and main results}

Recently, the present author originally considered the uniqueness of entire function sharing finite values with its differential-difference operators. Unfortunately, there was an error in the proof of [1, Theorem 1] (that is the equality (3.57) is wrong) and so there exists another case in the conclusion of [1, Theorem 1]. Here we correct it as follows.

Theorem 1. Let $f(z)$ be a transcendental entire function of finite order, let $\eta \neq 0$ be a finite complex number, $n \geq 1, k \geq 0$ two integers and let ab be two distinct finite complex values. If $f(z)$ and $\left(\Delta_{\eta}^{n} f(z)\right)^{(k)}$ share a CM and share $b$ IM, then either $f(z) \equiv\left(\Delta_{\eta}^{n} f(z)\right)^{(k)}$ or $a=2 b$,

$$
f(z) \equiv b e^{2(c z+d)}-2 b e^{c z+d}+2 b,
$$

The original article can be found online at https://doi.org/10.1007/s00025-021-01461-y. 
and

$$
\left(\Delta_{\eta}^{n} f(z)\right)^{(k)} \equiv b e^{c z}
$$

where $c=(-2)^{-\frac{n+1}{k}}$ for $k \geq 1$ and $d$ are two finite constants.

When $k=0$, the Corollary 1 in [1] is true.

\section{The Proof of Theorem 1}

We only consider the case $a b \neq 0$. From the proof of Theorem 1 as in [1], we know that

$$
\begin{aligned}
a & =2 b, \\
f(z) & =b e^{2(c z+d)}-2 b e^{c z+d}+2 b,
\end{aligned}
$$

and

$$
\left(\Delta_{\eta}^{n} f\right)^{(k)}(z)=b e^{c z+d},
$$

where $c \neq 0$ and $d$ are two finite constants. Then by (2.2) we have

$$
\left(\Delta_{\eta}^{n} f\right)^{(k)}(z)=b(2 c)^{k}\left(e^{2 c \eta}-1\right)^{n} e^{2(c z+d)}-2 b c^{k}\left(e^{c \eta}-1\right)^{n} e^{c z+d} .
$$

By (2.3) and (2.4), we claim that $e^{2 c \eta}-1=0$. Otherwise, we set $B=e^{2 c \eta}-1$ is a finite constant. Then by (2.4) and Lemma 2.8 in [1], we can obtain

$$
T\left(r,\left(\Delta_{\eta}^{n} f\right)^{(k)}(z)\right)=2 T\left(r, e^{c z+d}\right)+S\left(r, e^{c z+d}\right) .
$$

But we can know from (2.3) that

$$
T\left(r,\left(\Delta_{\eta}^{n} f\right)^{(k)}(z)\right)=T\left(r, e^{c z+d}\right)+S\left(r, e^{c z+d}\right),
$$

it follows from (2.5) that

$$
T\left(r,\left(\Delta_{\eta}^{n} f\right)^{(k)}(z)\right)=S\left(r, e^{c z+d}\right) .
$$

Since $f(z)$ and $\left(\Delta_{\eta}^{n} f\right)^{(k)}(z)$ share $a \mathrm{CM}$, and $b$ IM, and $f(z)$ is an entire function, then by the Second Nevanlinna's Fundamental Theorem and (2.7), we have

$$
\begin{aligned}
& T(r, f(z)) \leq \bar{N}(r, f(z))+\bar{N}\left(r, \frac{1}{f(z)-a}\right)+\bar{N}\left(r, \frac{1}{f(z)-b}\right)+S(r, f) \\
& =\bar{N}\left(r, \frac{1}{\left(\Delta_{\eta}^{n} f\right)^{(k)}(z)-a}\right)+\bar{N}\left(r, \frac{1}{\left(\Delta_{\eta}^{n} f\right)(k)(z)-b}\right)+S(r, f) \\
& \leq 2 T\left(r,\left(\Delta_{\eta}^{n} f\right)^{(k)}(z)\right)+S\left(r, e^{c z+d}\right)=S\left(r, e^{c z+d}\right),
\end{aligned}
$$


which implies $T(r, f(z))=S\left(r, e^{c z+d}\right)$, a contradiction. Therefore, $e^{2 c \eta}-1=0$. We slso claim that $e^{c \eta}=-1$. Otherwise, by (2.4) we obtain $\left(\Delta_{\eta}^{n l} f\right)^{(k)}(z) \equiv 0$, but it is impossible. Therefore, $e^{c \eta}=-1$ and we can get

$$
\left(\Delta_{\eta}^{n} f\right)^{(k)}(z)=(-2)^{n+1} c^{k} b e^{c z+d} \text {. }
$$

So we combine (2.3) and (2.5), we can get $c=(-2)^{-\frac{n+1}{k}}$ for $k \geq 1$. When $k=0$, we can see from $(2.3)$ and $(2.5)$ that $(-2)^{n+1}=1$, a contradiction.

This completes the proof of Theorem 1.

\section{Reference}

[1] Huang, X.H.: Unicity on entire function concerning its differential-difference operators. Results Math. 76, Article number: 147 (2021)

XiaoHuang Huang

Department of Mathematics

Sun Yat-sen University

Guangzhou 510275

P.R. China

e-mail: $1838394005 @ q q . c o m$

Publisher's Note Springer Nature remains neutral with regard to jurisdictional claims in published maps and institutional affiliations. 